Check for updates

Cite this: RSC Adv., 2019, 9, 15177

\title{
Mixture of quantum dots and ZnS nanoparticles as emissive layer for improved quantum dots light emitting diodes $\uparrow$
}

\author{
Taeyoung Song, Jun Young Cheong, Hyunjin Cho, Il-Doo Kim (iD) \\ and Duk Young Jeon (D) *
}

Recently, quantum dots based light-emitting diodes (QLEDs) have received huge attention due to the properties of quantum dots (QDs), such as high photoluminescence quantum yield (PLQY) and narrow emission. To improve the performance of QLEDs, reducing non-radiative energy transfer is critical. So far, most conventional methods required additional chemical treatment like giant shell and/or ligands exchange. However that triggers unsought shifted emission or reduced PLQY of QDs. In this work, we have firstly suggested a novel approach to improve the efficiency of QLEDs by introducing inorganic nanoparticles (NPs) spacer between QDs, without additional chemical treatment. As ZnS NPs formed a mixture layer with QDs, the energy transfer was reduced and the distance between the QDs increased, leading to improved PLQY of mixture layer. As a result, current efficiency (CE) of the QLED device was improved by twice compared with one using only QDs layer. This is an early report on utilizing ZnS NPs as an efficient spacer, which can be utilized to other compositions of QDs.

Received 26th February 2019

Accepted 4th May 2019

DOI: $10.1039 / c 9 r a 01462 d$

rsc.li/rsc-advances which is in contrast to QDs dispersed in non-polar solution. The EML structure induces energy transfer among QDs, including Förster resonant energy transfer (FRET). ${ }^{13,22-24}$ FRET is a nonradiative recombination process that limits the efficiency of devices. ${ }^{18-21}$

Thus far, to reduce non-radiative energy transfer, the distance between QD cores has been engineered according to shell thickness control and/or exchange of ligands with various lengths. ${ }^{18,21,23,24}$ However, such methods are accompanied by additional chemical treatment of the original QDs. In the case of shell thickness control, the high temperature that is required for shell growth triggers diffusion of ions in QDs. This leads to a shifted photoluminescence (PL) peak and reduced PLQY of QDs. In addition, the ligand exchange processes decrease the PLQY of QDs. ${ }^{25-27}$ Therefore, any methods of longer distance between QDs are needed for higher efficiency of QLEDs, except for additional chemical treatments of QDs.

Herein, we firstly suggest that the efficiency of QLED devices is improved using an inorganic NP spacer between QDs without additional chemical treatment of the QDs. Inorganic nanoparticles (NPs) consist of ZnS that is generally used for the shell of type-I core/shell heterostructured QDs because it has a larger bandgap than the core of the QDs $(\sim 3.6 \mathrm{eV}) .{ }^{28}$ In order to apply ZnS NPs to the EML of QLEDs, we mixed ZnS NPs with greenemitting CdZnSeS/ZnS QDs dispersed in non-polar solvent. The solution was then applied to the EML of QLEDs by simple spin-casting.

The formed mixture layers of the QDs and ZnS NPs showed that the non-radiative energy transfer was reduced due to $\mathrm{ZnS}$
Department of Materials Science and Engineering, Korea Advanced Institute of Science and Technology, 291 Daehak-ro, Yuseong-gu, Daejeon 305-701, Republic of Korea. E-mail:dyjeon@kaist.ac.kr

$\dagger$ Electronic supplementary information (ESI) available. See DOI: 10.1039/c9ra01462d 
NPs acting as spacers increasing the distance between the QDs. ${ }^{29-31}$ Consequently, the PLQY of the mixture layers was improved with increasing ratio of ZnS NPs, and the current efficiency (CE) of the QLED device with the mixture layer was enhanced by about two times compared with only the QD layer. This simple structure would be applicable to not only CdZnSeS/ ZnS QDs but also to QDs with various compositions due to the large bandgap of ZnS NPs. ${ }^{31,32}$

\section{Experimental}

\section{Materials}

Oleic acid (OA, 90\%), 1-octadecene (1-ODE, 90\%), trioctylphosphine (TOP, 97\%), zinc acetate (99.99\%), sulfur (99.998\%), zinc diethyldithiocarbamate (Zn DDTC, 97\%), ZnO (99.999\%), cadmium acetate $(99.995 \%)$, Se powder $(99.99 \%)$ zinc acetate dihydrate $(\geq 98 \%)$, lithium hydroxide monohydrate $(\geq 98 \%)$, and poly[ $(9,9-$ dioctylfluorenyl-2,7-diyl)-co-(4,4'-( $N$-(4-sec-butylphenyl)diphenylamine))] (TFB) were purchased from Sigma Aldrich.

\section{Synthesis of green-emitting CdZnSeS/ZnS quantum dots}

Although the band-edge emission of the QDs can be changed by using only the size effect, the quantum dots have possibility of low photoluminescence efficiency or low stability due to increased surface to volume ratio. Hence, we adopted the CdZnSeS/ZnS quantum dots of alloy structure containing additional $\mathrm{Zn}$ and $\mathrm{S}^{33,34}$ The synthetic method of the QDs is as follows. $277 \mathrm{mg}$ of $\mathrm{ZnO}, 32 \mathrm{mg}$ of cadmium acetate, $15 \mathrm{~mL}$ of 1ODE and $7 \mathrm{~mL}$ of OA were loaded in three-neck flask and heated to $130{ }^{\circ} \mathrm{C}$ for $1 \mathrm{~h}$ under high vacuum. Subsequently, after increasing temperature to $320{ }^{\circ} \mathrm{C}$ for colorless and clear solution under Ar atmosphere, the solution was cooled to $300{ }^{\circ} \mathrm{C}$. Then $158 \mathrm{mg}$ of selenium and $64 \mathrm{mg}$ of sulfur powder dissolved in $2 \mathrm{~mL}$ of TOP was quickly injected into the flask. After $10 \mathrm{~min}$, the flask was cooled to $225^{\circ} \mathrm{C}$ and $200 \mathrm{mg}$ of Zn DDTC dissolved in $2 \mathrm{~mL}$ of TOP was injected into the solution. Next, the solution was heated to $280{ }^{\circ} \mathrm{C}$ and maintained for $30 \mathrm{~min}$.

\section{Synthesis of ZnS NPs}

$289 \mathrm{mg}$ of sulfur, $219 \mathrm{mg}$ of zinc acetate, $6 \mathrm{~mL}$ of OA, and $6 \mathrm{~mL}$ of 1 ODE were loaded into $100 \mathrm{~mL}$ three-neck flask. After being degassed under high vacuum for $1 \mathrm{~h}$ at $50{ }^{\circ} \mathrm{C}$, the solution was heated to $300{ }^{\circ} \mathrm{C}$ and maintained for $60 \mathrm{~min}$ under $\mathrm{Ar}$ atmosphere.

\section{Synthesis of ZnO NPs}

ZnO NPs were synthesized based on a previous paper. ${ }^{35} 439 \mathrm{mg}$ of zinc acetate dihydrate was added in $20 \mathrm{~mL}$ of absolute ethanol and the solution was heated to $80^{\circ} \mathrm{C}$ for $1 \mathrm{~h}$ with stirring. After homogeneous and transparent solution was obtained, $118 \mathrm{mg}$ of lithium hydroxide monohydrate was added and the solution was sonicated for $1 \mathrm{~h}$ to obtain ZnO NPs.

\section{Device fabrication}

Patterned ITO glass substrates were sonicated in three different solvents (acetone, methanol and isopropyl alcohol) for $30 \mathrm{~min}$ and dried at $120{ }^{\circ} \mathrm{C}$ in oven and then treated with UV-ozone for $10 \mathrm{~min}$. Poly(3,4-ethylenedioxythiophene):poly-(styrenesulfonate) (PEDOT:PSS, Clevios P VP AI4083) was spun-cast on the ITO substrate with a spin-rate of $3000 \mathrm{rpm}$ for $45 \mathrm{~s}$, followed by baking at $180{ }^{\circ} \mathrm{C}$ for $1 \mathrm{~h}$. TFB layers were casted on the PEDOT:PSS from solution ( $8 \mathrm{mg} \mathrm{mL}^{-1}$ in $p$-xylene) with spin-rate of $3000 \mathrm{rpm}$ for $45 \mathrm{~s}$ and then annealed at $180^{\circ} \mathrm{C}$ for $1 \mathrm{~h}$. The mixture solutions of QDs and ZnS NPs (20 mg mL $\mathrm{mL}^{-1}$ in octane) were spun-cast directly on TFB layer with spin-rate of $3000 \mathrm{rpm}$ for $45 \mathrm{~s}$ and dried at $100{ }^{\circ} \mathrm{C}$ for $30 \mathrm{~min}$. On the mixture layers, the ZnO NPs $(40 \mathrm{mg}$ $\mathrm{mL}^{-1}$ in 2-methoxyethanol) were formed with spin rate of $2000 \mathrm{rpm}$ for $45 \mathrm{~s}$. Finally, Al cathode layer of $120 \mathrm{~nm}$ thickness was deposited by thermal evaporation at base pressure of $1 \times$ $10^{-7}$ torr for complete devices.

\section{Characterization}

Morphological features were obtained using atomic force microscope (AFM, Innova-LabRam HR 800, Bruker and Horiba), scanning electron microscope (SEM, SU8230, Hitachi) and field emission transmission electron microscopy (TE-TEM, Tecnai G2 F30 S-Twin). Energy dispersive X-ray spectroscopy (EDS) mapping images were observed using SEM with EDS. Fourier transform infrared (FTIR) spectroscopy, thermogravimetric (TG) analysis and X-ray fluorescence (XRF) analysis were conducted using Nicolet iS50 of Thermo Fisher Scientific Instrument, TG209F1 Libra of Netzsch, and ZSX Primus II of Rigaku, respectively. The photoluminescence spectra were recorded using FL920, Edinburgh instruments, and F-7000, Hitachi. Xray diffraction (XRD) patterns were obtained by a X-ray diffractometer (SmartLab, Rigaku). The decay times of photoluminescence were recorded by FL920 with picosecond pulsed diode laser of $470 \mathrm{~nm}$, EPL-470, and the values were calculated using a F900 program. The decay times of layers were measured at $528.2 \mathrm{~nm}$ at room temperature. For the QLED, electron only and hole only devices, the current-luminance versus driving voltage results were obtained by Konica Minolta spectroradiometer (CS-2000) and a Sourcemeter (Keithley 2635A). X-Ray photoelectron spectroscopy (XPS) data and ultraviolet photoemission spectroscopy (UPS) spectra were obtained using Kalpha (Thermo VG scientific) and Axis-Supra (Kratos), respectively.

\section{Results and discussion}

Fig. 1a and b show high-resolution TEM images of the synthesized ZnS NPs and CdZnSeS/ZnS QDs, respectively. Lowresolution TEM images of ZnS NPs and QDs are shown in Fig. S1. $\dagger$ In Fig. 1d, the XRD pattern shows that the ZnS NPs are a zinc blende structure (ICDD no 01-077-3378), and the selected area electron diffraction (SAED) pattern also indicates that the ZnS NPs possess a zinc blende structure and high crystallinity (Fig. 1a, inset). Additionally, the SAED pattern shows that three out of five diffraction rings near the centre were indexed to the same positions as (111), (220), (311). Fig. 1a shows that the ZnS NPs have lattice fringe spacing parallel to $\sim 0.31 \mathrm{~nm}$ and this was assigned to the d-spacing of (111) plane of zinc blende 

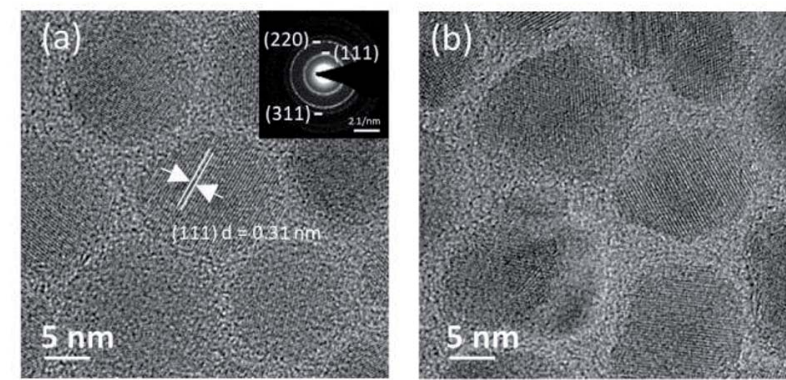

(c)

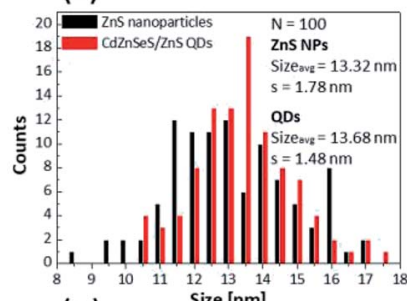

(e)

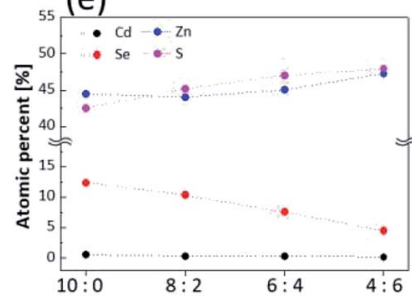

(d)

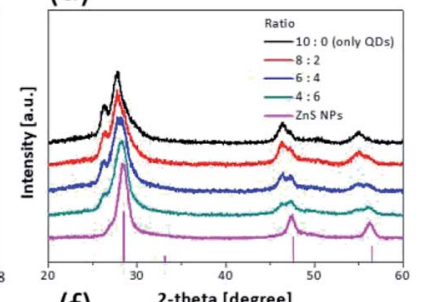

$(\mathrm{f})$

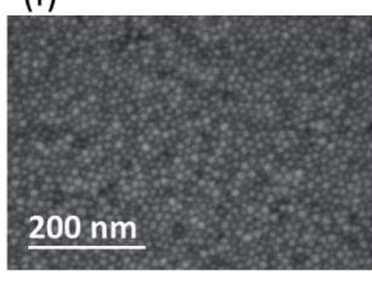

in the synthesized ZnS NPs and this result is similar to that obtained by TG analysis (Table S1 $\dagger$ ). The ZnS NPs and QDs would be dispersed in the same non-polar solvent.

Octane was chosen as the solvent to analyse the characteristics of the mixture of the QDs and ZnS NPs because it has already been used as an orthogonal solvent for quality improvement of EML in QLEDs. ${ }^{40}$ The mixture layer was deposited by spin-casting of the solution mixed with the QDs and ZnS NPs. Solution concentration was controlled to $20 \mathrm{mg}$ $\mathrm{mL}^{-1}$ and ratio of the QDs to ZnS NPs was set to $10: 0$ (only QDs), $8: 2,6: 4$, and $4: 6$ according to weight percent of these. Fig. 1d shows the XRD patterns of the mixture layers coated on glass with different ratios. When the layer of 10:0 was formed by only QDs, Fig. 1d shows that the XRD peaks gradually shifted to peaks assigned to the $\mathrm{ZnS}$ structure, and it was confirmed that the mixture layers were well blended in terms of macroanalysis of the XRD. SEM-EDS mapping images also show uniform distribution of the QDs and ZnS NPs for the layer of $6: 4$ (Fig. S5 $\dagger$ ). XPS analysis showed that the proportion of cadmium and selenium decreased contrary to increasing proportion of zinc to sulfur depending on increasing the ratio of ZnS NPs (Fig. 1e). The detailed data of atomic percentages are shown in Table S2.†

In Fig. 1f, the SEM image shows the uniform surface of the mixture layer of $6: 4$. Although the layer consists of two different materials, it was identified that inhomogeneous aggregations of each particle were not observed in the other layers as well as the layer of $6: 4$ (Fig. S6†). In addition, the surface roughness of the layers became smoother after mixing. of the QDs with the ZnS NPs because the QDs were mixed with smaller sized-ZnS NPs that fill empty space in the QD layer (Fig. S7 $\dagger$ ). This means that the uniform mixture layer is more adequate to apply it as the EML of QLEDs for which the layer quality is important. ${ }^{41-43}$

The PL properties of each mixture layer are shown in Fig. 2

ZnS. ${ }^{36}$ Fig. 1d also shows the XRD pattern of the synthesized green-emitting CdZnSeS/ZnS QDs at 10:0 (only QDs). The detailed XRD pattern of the QDs, which have an alloy core structure and ZnS shell structures, is shown in Fig. S2. $\dagger$

The average sizes of the ZnS NPs and QDs were $13.32 \mathrm{~nm}$ and $13.68 \mathrm{~nm}$, respectively (Fig. 1c). The synthesized ZnS NPs were similar to QDs with about 3\% difference to exclude variables for size and capping ligands. The role of a ligand is to remove surface defects and improve colloidal dispersion stability in solvent. The synthesized ZnS NPs were capped with the same oleic acid ligand that was used for the green-emitting CdZnSeS/ ZnS QDs. ${ }^{37}$ In Fig. S3, $\dagger$ the $\mathrm{C}-\mathrm{H}$ vibrations of the $\mathrm{CH}_{2}$ group and the $\mathrm{COO}^{-}$vibrations of the carboxylic group, which belong to the oleic acid ligand, were confirmed using FTIR spectroscopy. It was identified that the carboxylate anion formed chemical bond with the $\mathrm{Zn}$ cation on the surface. ${ }^{38,39}$ Organic ligand materials have a lower evaporation temperature than inorganic ones, and TG analysis was conducted for checking the proportion of ZnS NP ligands. At temperature over $450{ }^{\circ} \mathrm{C}$, all of the organic ligands were evaporated, and the content was about $16 \%$, which is similar to that of the QD ligands (Fig. S4 $\dagger$ ). In addition, XRF analysis showed about $13 \%$ of the carbon content

and Table 1. The layer of 10:0 (only QDs) exhibited a redshifted PL peak (535.3 nm) and reduced PLQY (34.3\%) compared with the solution state of the QDs (528.2 nm, 72.5\%) (Table 1). In the film state, a factor of the changed properties is non-radiative FRET between QDs. The FRET vigorously occurs by interdot coupling strength when the distance between the donor and the acceptor becomes closer, and spectra overlap of the donor emission and the acceptor absorption becomes greater..$^{22-24,30,31}$ Because the synthesized QDs have considerably large aforementioned overlap of the spectra due to small Stoke's (a)

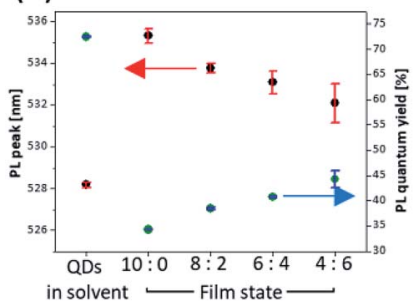

(b)

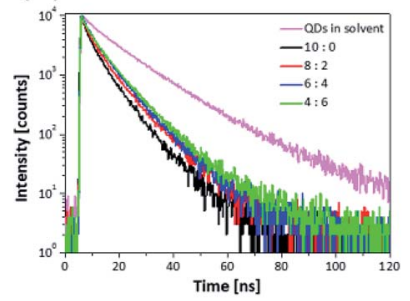

Fig. 2 (a) PL peaks, PLQYs and (b) transient PL decay curves corresponding to four different ratios of the QDs to the ZnS NPs. 
Table 1 Details on the PLQYs, PL peaks and decay times demonstrated in Fig. 2

\begin{tabular}{llll}
\hline & PLQY [\%] & PL peak [nm] & Decay time [ns] \\
\hline QDs in solution & 72.5 & 528.2 & 13.9 \\
$10: 0$ & 34.3 & 535.3 & 6.1 \\
$8: 2$ & 38.5 & 533.8 & 6.8 \\
$6: 4$ & 40.8 & 533.1 & 7.1 \\
$4: 6$ & 44.3 & 532.1 & 7.8
\end{tabular}

shift as shown in Fig. 3a. When the QDs are close to each other, the large spectral overlap gives rise to not radiative recombination of excitons formed in the QDs but excitation of other QDs in close proximity and non-radiative quenching of excitons. ${ }^{8}$ Consequently, the FRET is accompanied by the redshifted PL peaks and reduced PLQYs in the QD layer where the distance between QDs is extremely short compared with that in the solution state. Although the FRET exists because of the intrinsic characteristics of the QDs, it should be eliminated for improved performance of QLED device. However, depending on increasing ratio of ZnS NPs, the PL peak and the PLQY gradually were recovered, and then the layer of $4: 6$ had a PL peak of $532.1 \mathrm{~nm}$ and PLQY of $44 \%$ (Fig. 2a). These properties show that the non-radiative recombination of energy transfer decreases due to presence of the ZnS NPs. Fig. 3b shows a schematic illustration of the role of the ZnS NPs. Because the large band gap of ZnS NPs starts to absorb wavelength below $350 \mathrm{~nm}$, it is hard to absorb light emitted from the QDs. Hence the aforementioned spectral overlap decreases (Fig. 3a). ${ }^{31,44}$ In addition, as the ZnS NPs act as spacers increasing the distance between QDs, the ZnS NPs support the radiative recombination of excitons formed in QDs. In other words, the factors of suppressing FRET become favourable for the improved QLED performance due to using the mixture layer between the $\mathrm{ZnS}$ nanoparticles and the quantum dots. This also means that because the absorption region of ZnS NPs is in the UV range, the simple method would be sufficiently extended not only to the green-emitting CdZnSeS/ZnS QDs but also to other QDs emitting visible light. ${ }^{32}$

Fig. $2 \mathrm{~b}$ shows the decay time of films corresponding to the ratio of the QDs to ZnS NPs. The QD solution has 13.9 ns of decay time. However, the decay time of the layer of $10: 0$ drastically decreased to $6.1 \mathrm{~ns}$ by the aforementioned energy transfer, which is a faster reaction than the radiative
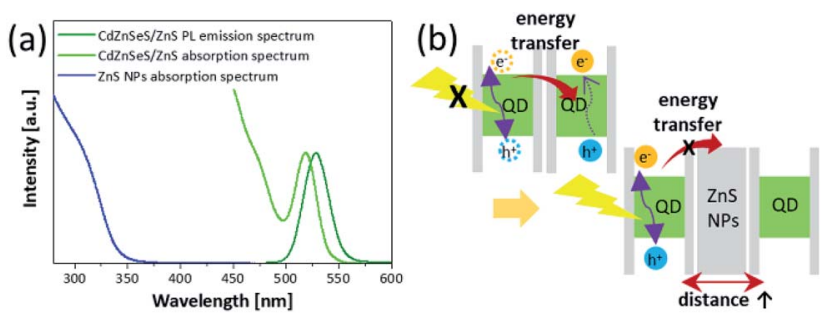

Fig. 3 (a) PL and absorption spectra of the QDs and absorption spectrum of the ZnS NPs. (b) Schematic illustration on the ZnS NPs that block the energy transfer. recombination rate of excitons in QDs. ${ }^{22,23,45}$ However, as the ZnS NP content of the mixture layer increased, reduced decay time was recovered toward the solution state, and the $4: 6$ layer had $7.8 \mathrm{~ns}$ of decay time. Additionally, the energy transfer efficiency is calculated from using eqn (1). ${ }^{46}$ The energy transfer efficiency $\left(\eta_{\text {FRET }}\right)$ is

$$
\eta_{\mathrm{FRET}}=1-\frac{\tau_{\mathrm{DA}}}{\tau_{\mathrm{D}}}
$$

calculated by two factors, which are decay time $\left(\tau_{\mathrm{DA}}\right)$ of donors where are together with acceptors and a decay time $\left(\tau_{\mathrm{D}}\right)$ of donors without acceptors (Table S3 $\uparrow$ ). In this experiment, the $\tau_{\mathrm{DA}}$ was calculated by a decay time of film states and the $\tau_{\mathrm{D}}$ was calculated for QDs in solution. It was assumed that the energy transfer between QDs did not occur in solution due to much longer distance. Consequently, the energy transfer decreased from $56.1 \%$ to $43.9 \%$ at the layer of $4: 6$ compared with the layer of $10: 0$, meaning the ZnS NPs were effective in eliminating energy transfer.

In order to apply the mixture layer as an EML of QLED, device performance was investigated using solution-processed QLEDs structure. This device adopted a normal structure and was fabricated by sequential deposition of PEDOT:PSS, TFB, the mixture layers, ZnO NPs, and finally an Al cathode on an ITO (anode) substrate. Table 2 summarizes the device performance of the QLEDs. Fig. 4a shows the architecture of the devices and corresponding energy levels. Fig. $4 \mathrm{~b}$ and c show the current density-voltage-luminance $(J-V-L)$ characteristics and $\mathrm{CE}$ depending on voltage for the QLEDs, respectively. Fig. 4d exhibits graphs of CE vs. luminance for QLEDs depending on the ratio of QDs to ZnS NPs.

Based on the results, devices incorporating the ZnS NPs exhibit significantly improved maximum CE. Specifically, the QLED of 6:4 showed a peak CE of $8.10 \mathrm{~cd} \mathrm{~A}^{-1}$, which is about twice compared with the QLED of $10: 0$. In addition, at the same luminance condition, all of the performances for QLEDs consisting of a mixture layer were higher than QLED consisting of only a QD layer. At $1000 \mathrm{~cd} \mathrm{~cm}^{-2}$, the QLED of $8: 2$ and $4: 6$ had CEs of $1.21 \mathrm{~cd} \mathrm{~A}^{-1}$ and $1.45 \mathrm{~cd} \mathrm{~A}^{-1}$, respectively. Notably, the QLED of $6: 4$ had $\mathrm{CE}$ of $2.06 \mathrm{~cd} \mathrm{~A}^{-1}$, which is about twice that of the QLED of $10: 0\left(0.91 \mathrm{~cd} \mathrm{~A}^{-1}\right)$. The improved results are attributed to the ZnS NPs. Although the maximum luminance of QLEDs including ZnS NPs was improved, the QLED of $4: 6$ showed decreased maximum luminance $\left(12401 \mathrm{~cd} \mathrm{~m}^{-2}\right)$ compared with the QLED of $6: 4$ (Fig. $4 \mathrm{~d}$ ). This is because the absolute quantity of QDs decreased as the content of ZnS NPs increased in the mixture layers.

Fig. $4 \mathrm{~b}$ shows that the current density increased and the turn-on voltage $\left(V_{\text {turn-on }}\right)$ decreased corresponding to the content of ZnS NPs. Table 2 shows that although the $V_{\text {turn-on }}$ was $3.7 \mathrm{~V}$ for the QLED of $10: 0$, it gradually shifted to $2.7 \mathrm{~V}$ for the QLED of $4: 6$. In addition, the voltages assigned to the CE peaks became lower (Fig. 4c). The CE peak that was $4.04 \mathrm{~cd} \mathrm{~A}^{-1}$ at $6.5 \mathrm{~V}$ for the QLED of $10: 0$ shifted to $5.23 \mathrm{~cd} \mathrm{~A}^{-1}$ at $5.25 \mathrm{~V}$ for the QLED of $4: 6$. This means that the ZnS NPs in the EML act not only spacers but also charge injection materials that transfer charges to QDs well. In order to identify the charges injection 
Table 2 Device performances of the QLEDs using the mixture layer as an EML

\begin{tabular}{llll}
\hline & \multicolumn{2}{c}{ Current efficiency $\left[\mathrm{cd} \mathrm{A}^{-1}\right]$} & \\
\cline { 2 - 3 } & @1000 cd cm & & \\
& & Max. luminance $\left[\mathrm{cd} \mathrm{m}^{-2}\right]$ & 14814 \\
$10: 0$ & 0.91 & $4.04 @ 6.5 \mathrm{~V}$ & 18064 \\
$8: 2$ & 1.21 & 5.14 @ $6.25 \mathrm{~V}$ & 21432 \\
$6: 4$ & 2.06 & 8.10 @ $5.5 \mathrm{~V}$ & 12401 \\
$4: 6$ & 1.45 & 5.23 @ $5.25 \mathrm{~V}$ & \\
${ }^{a} V_{\text {trun-on }}$ is defined at the luminance of $1 \mathrm{~cd} \mathrm{~m}^{-2}$. & &
\end{tabular}

(a)

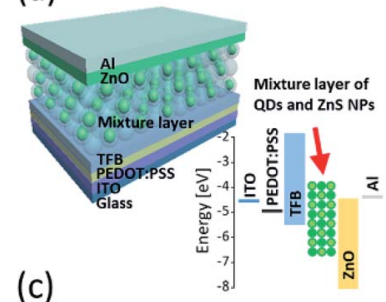

(c)

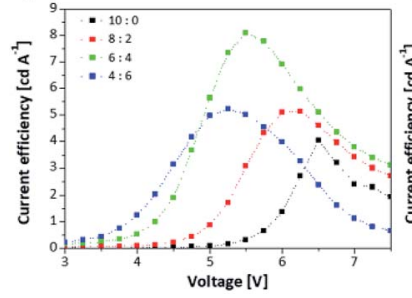

(b)

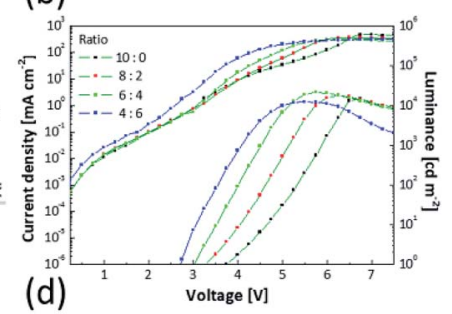

(d)

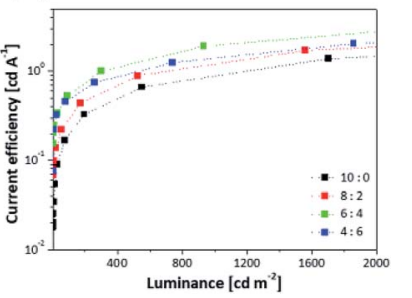

Fig. 4 (a) Schematic device structure and energy level diagram. (b) J$V-L$ characteristics of QLEDs with different ratios of the QDs to the ZnS NPs. CE as a function of (c) voltage and (d) luminance for the QLEDS.

properties of ZnS NPs, an electron only device (EOD) and a hole only device (HOD) were fabricated. The EOD was fabricated by sequential deposition of $\mathrm{Al}$, mixture layers, $\mathrm{ZnO}$, and $\mathrm{Al}$ to move the electrons except for holes, and the HOD was fabricated by deposition of PEDOT:PSS, TFB, mixture layers, $\mathrm{MoO}_{3}$, and $\mathrm{Al}$ electrode for moving only holes. The energy level structures of EOD and HOD are shown in Fig. S8. $\dagger$ In Fig. 5, at low voltage range, due to insufficient energy for crossing the energy barrier of layers, the current density doesn't consist of injected charges from electrode but most charges existing inside layers of device. This region is governed by Ohm's law and called ohmic

(a)

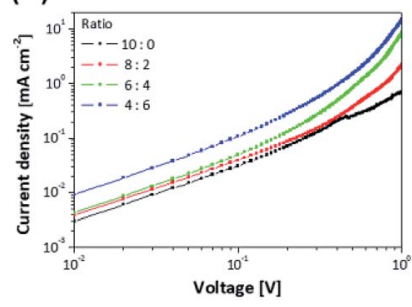

(b)

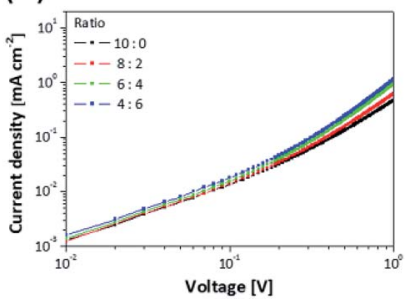

Fig. 5 Current density-voltage graphs for (a) the EOD (Al/mixture layer/ZnO/Al) and (b) the HOD (ITO/PEDOT:PSS/TFB/mixture layer/ $\mathrm{MoO}_{3} / \mathrm{Al}$ ) corresponding to ratio of the QDs to the ZnS NPs.

region. ${ }^{15,47}$ In the ohmic region of $\log J-\log V$ curves, the current density is proportional to the voltage and has linear relationship $(J \propto V)$. As the voltage increases, a region where slope of the current density increases appears, and it is called space chargelimited current region. The current density of the region behaves with Mott-Gurney law and is dramatically increased by added charges from electrode. ${ }^{\mathbf{4 8 , 4 9}}$

The EOD has a relatively larger current density than the HOD due to the difference of energy barrier between each charge transport layer to the QDs. ${ }^{15}$ Hence, Fig. 5a and b show that the EOD of $10: 0$ has a larger current density $\left(0.031 \mathrm{~mA} \mathrm{~cm}^{-2}\right.$ at 0.1 $\mathrm{V})$ compared with the HOD of $10: 0\left(0.013 \mathrm{~mA} \mathrm{~cm}^{-2}\right)$. Fig. S9 $\dagger$ shows that although high occupied molecular orbital levels of mixture layer shifted to a deep level depending on increasing the ZnS NP portion, the current density gradually increased. In terms of charge mobility, the layers containing ZnS NPs have a higher mobility than only the QD layer due to the difference in energy levels of the core/shell heterostructure of QDs. This is because the ZnS shell of QDs has a larger bandgap than the core and acts as an energy barrier disturbing the movement of charges. $^{\mathbf{1 2 , 5 0 , 5 1}}$ Additionally, the current density of EOD was relatively more improved than that of HOD depending on the amount of contained ZnS NPs because the zinc blende structure of $\mathrm{ZnS}$ has an electron mobility of $180 \mathrm{~cm}^{2} \mathrm{~V}^{-1} \mathrm{~s}^{-1}$, which is more 36 times than a hole mobility $\left(5 \mathrm{~cm}^{2} \mathrm{~V}^{-1} \mathrm{~s}^{-1}\right)$ at $300 \mathrm{~K} .^{52,53}$ Based on the results, as the amount of ZnS NPs increased, the difference between the current densities of electrons and holes became bigger, and this induced the imbalanced charge injection to the QDs layer. Although the improved properties of charge injection contributed to low $V_{\text {turn-on, the imbalance }}$ increased. Hence, the QLED of 4:6 having a low absolute amount of QDs had lower CE compared with the other QLEDs incorporating ZnS NPs. This increment of current density affected the leakage current and current density of $J-V-L$ curves (Fig. 4b).

\section{Conclusions}

We successfully utilized inorganic ZnS NP as a spacer between QDs, which is different from the conventional approach of controlling the shell thickness and/or ligands of varying lengths. The introduction of ZnS NPs increased the PLQY of the mixture layers because ZnS NPs decreased the non-radiative recombination generated by energy transfer. In terms of the EML of QLEDs, the QLED consisting of the layer of $6: 4$ showed 
$8.10 \mathrm{~cd} \mathrm{~A}^{-1}$ of $\mathrm{CE}$, which was two times higher than that of the QLED consisting of only a QD layer. The results suggest that inorganic ZnS NPs can be used as effective spacers to enhance the performance of QLEDs, which can also be extended to other QDs with different compositions due to a large band gap.

\section{Conflicts of interest}

There are no conflicts to declare.

\section{Acknowledgements}

This research was supported by the Creative Materials Discovery Program through the National Research Foundation of Korea (NRF) funded by the Ministry of Science, ICT and Future Planning (NRF-2016M3D1A1900035).

\section{References}

1 L.-M. Yang, V. Bačić, I. A. Popov, A. I. Boldyrev, T. Heine, T. Frauenheim and E. Ganz, J. Am. Chem. Soc., 2015, 137, 2757-2762.

2 L. M. Yang, E. Ganz, Z. Chen, Z. X. Wang and P. v. R. Schleyer, Angew. Chem., Int. Ed., 2015, 54, 9468-9501.

3 B. Song, Y. Zhou, H.-M. Yang, J.-H. Liao, L.-M. Yang, X.-B. Yang and E. Ganz, J. Am. Chem. Soc., 2019, 141, 36303640 .

4 L.-M. Yang, I. A. Popov, A. I. Boldyrev, T. Heine, T. Frauenheim and E. Ganz, Phys. Chem. Chem. Phys., 2015, 17, 17545-17551.

5 L.-M. Yang, I. A. Popov, T. Frauenheim, A. I. Boldyrev, T. Heine, V. Bačić and E. Ganz, Phys. Chem. Chem. Phys, 2015, 17, 26043-26048.

6 L.-M. Yang and E. Ganz, Phys. Chem. Chem. Phys., 2016, 18, 17586-17591.

7 A. P. Alivisatos, Science, 1996, 271, 933-937.

8 O. I. Mićić, J. Sprague, Z. Lu and A. J. Nozik, Appl. Phys. Lett., 1996, 68, 3150-3152.

9 P. Reiss, J. Bleuse and A. Pron, Nano Lett., 2002, 2, 781-784.

10 L. Li, T. J. Daou, I. Texier, T. T. Kim Chi, N. Q. Liem and P. Reiss, Chem. Mater., 2009, 21, 2422-2429.

11 O. Chen, J. Zhao, V. P. Chauhan, J. Cui, C. Wong, D. K. Harris, H. Wei, H.-S. Han, D. Fukumura and R. K. Jain, Nat. Mater., 2013, 12, 445.

12 P. Reiss, M. Protiere and L. Li, Small, 2009, 5, 154-168.

13 B. S. Mashford, M. Stevenson, Z. Popovic, C. Hamilton, Z. Zhou, C. Breen, J. Steckel, V. Bulovic, M. Bawendi and S. Coe-Sullivan, Nat. Photonics, 2013, 7, 407.

14 L. Qian, Y. Zheng, J. Xue and P. H. Holloway, Nat. Photonics, 2011, 5, 543.

15 X. Dai, Z. Zhang, Y. Jin, Y. Niu, H. Cao, X. Liang, L. Chen, J. Wang and X. Peng, Nature, 2014, 515, 96.

16 H. T. Nguyen, H. Jeong, J.-Y. Park, Y. Ahn and S. Lee, ACS Appl. Mater. Interfaces, 2014, 6, 7286-7291.

17 H.-M. Kim, S. Cho, J. Kim, H. Shin and J. Jang, ACS Appl. Mater. Interfaces, 2018, 10, 24028-24036.
18 J. Lim, B. G. Jeong, M. Park, J. K. Kim, J. M. Pietryga, Y. S. Park, V. I. Klimov, C. Lee, D. C. Lee and W. K. Bae, Adv. Mater., 2014, 26, 8034-8040.

19 K.-H. Lee, J.-H. Lee, H.-D. Kang, B. Park, Y. Kwon, H. Ko, C. Lee, J. Lee and H. Yang, ACS Nano, 2014, 8, 4893-4901.

20 L. Sun, J. J. Choi, D. Stachnik, A. C. Bartnik, B.-R. Hyun, G. G. Malliaras, T. Hanrath and F. W. Wise, Nat. Nanotechnol., 2012, 7, 369.

21 B. N. Pal, Y. Ghosh, S. Brovelli, R. Laocharoensuk, V. I. Klimov, J. A. Hollingsworth and H. Htoon, Nano Lett., 2011, 12, 331-336.

22 S. Crooker, J. Hollingsworth, S. Tretiak and V. I. Klimov, Phys. Rev. Lett., 2002, 89, 186802.

23 R. Koole, P. Liljeroth, C. de Mello Donegá, D. Vanmaekelbergh and A. Meijerink, J. Am. Chem. Soc., 2006, 128, 10436-10441.

24 J. J. Choi, J. Luria, B.-R. Hyun, A. C. Bartnik, L. Sun, Y.-F. Lim, J. A. Marohn, F. W. Wise and T. Hanrath, Nano Lett., 2010, 10, 1805-1811.

25 Y. su Kim, Y. Lee, Y. Kim, D. Kim, H. S. Choi, J. C. Park, Y. S. Nam and D. Y. Jeon, RSC Adv., 2017, 7, 10675-10682.

26 Y. Kim, H. S. Jang, H. Kim, S. Kim and D. Y. Jeon, ACS Appl. Mater. Interfaces, 2017, 9, 32097-32105.

27 S. Yu, X.-B. Fan, X. Wang, J. Li, Q. Zhang, A. Xia, S. Wei, L.-Z. Wu, Y. Zhou and G. R. Patzke, Nat. Commun., 2018, 9, 4009.

28 C.-T. Cheng, C.-Y. Chen, C.-W. Lai, W.-H. Liu, S.-C. Pu, P.-T. Chou, Y.-H. Chou and H.-T. Chiu, J. Mater. Chem., 2005, 15, 3409-3414.

29 A. R. Clapp, I. L. Medintz and H. Mattoussi, ChemPhysChem, 2006, 7, 47-57.

30 S. W. Clark, J. M. Harbold and F. W. Wise, J. Phys. Chem. C, 2007, 111, 7302-7305.

31 K. Chou and A. Dennis, Sensors, 2015, 15, 13288-13325.

32 A. L. Rogach, A. Eychmüller, S. G. Hickey and S. V. Kershaw, Small, 2007, 3, 536-557.

33 W. K. Bae, K. Char, H. Hur and S. Lee, Chem. Mater., 2008, 20, 531-539.

34 S. Jun and E. Jang, Angew. Chem., Int. Ed., 2013, 52, 679-682. 35 K. Kim, M. Suh, J. Choi, D. Lee, Y. Kim, S. H. Cheong, D. Kim and D. Y. Jeon, Adv. Funct. Mater., 2015, 25, 7450-7456.

36 H. Ahn and J. Lee, Mater. Lett., 2013, 106, 308-312.

37 M. Green, J. Mater. Chem., 2010, 20, 5797-5809.

38 B. Fritzinger, R. K. Capek, K. Lambert, J. C. Martins and Z. Hens, J. Am. Chem. Soc., 2010, 132, 10195-10201.

39 P. Tao, Y. Li, R. W. Siegel and L. S. Schadler, J. Mater. Chem. C, 2013, 1, 86-94.

40 Y. Zou, M. Ban, W. Cui, Q. Huang, C. Wu, J. Liu, H. Wu, T. Song and B. Sun, Adv. Funct. Mater., 2017, 27, 1603325.

41 D. Lee, D. Kim, Y. Lee and D. Y. Jeon, Nanotechnology, 2017, 28, 235201.

42 Y. Lee, M. Suh, D. Kim, D. Lee, H. Chang, H. S. Lee, Y. W. Kim, T. Kim, K. S. Suh and D. Y. Jeon, Adv. Funct. Mater., 2014, 24, 6465-6472.

43 D. Kim, Y. Fu, S. Kim, W. Lee, K.-H. Lee, H. K. Chung, H.-J. Lee, H. Yang and H. Chae, ACS Nano, 2017, 11, 19821990. 
44 L. Stryer, Annu. Rev. Biochem., 1978, 47, 819-846.

45 M. Achermann, M. A. Petruska, S. A. Crooker and V. I. Klimov, J. Phys. Chem. B, 2003, 107, 13782-13787.

46 E. Mutlugun, O. Samarskaya, T. Ozel, N. Cicek, N. Gaponik, A. Eychmüller and H. V. Demir, Opt. Express, 2010, 18, 10720-10730.

47 H. Meier, Monographs in modern chemistry, 1974.

48 N. F. Mott and R. W. Gurney, Electronic Processes in Ionic Crystals, Oxford University Press, Oxford, 1st edn, 1940.

49 R. Hikmet, D. Talapin and H. Weller, J. Appl. Phys., 2003, 93, 3509-3514.
50 P. R. Brown, D. Kim, R. R. Lunt, N. Zhao, M. G. Bawendi, J. C. Grossman and V. Bulovic, ACS Nano, 2014, 8, 58635872.

51 X. Yao, Z. Song, L. Mi, G. Li, X. Wang, X. Wang and Y. Jiang, Sol. Energy Mater. Sol. Cells, 2017, 164, 122-127.

52 R. C. Weast, M. J. Astle and W. H. Beyer, CRC handbook of chemistry and physics, CRC press Boca Raton, FL, 1988.

53 A. F. Wells, Structural inorganic chemistry, Oxford University Press, 2012. 